\title{
Marking 15 years of efforts towards a comprehensive European TB surveillance system: the epidemiological situation of TB in the EU/EEA in 2009
}

V Hollo (Vahur.Hollo@ecdc.europa.eu) ${ }^{1}$, P Zucs $^{1}$, C Ködmön¹, A Sandgren ${ }^{1}$, D Manissero¹

1. Tuberculosis Programme, European Centre for Disease Prevention and Control (ECDC), Stockholm, Sweden

Citation style for this article:

Hollo V, ZuCs P, Ködmön C, Sandgren A, Manissero D. Marking 15 years of efforts towards a comprehensive European TB surveillance system: the epidemiological situation of TB in the EU/EEA in 2009. Euro Surveill. 2011;16(12):pii=19822. Available online: http://www.eurosurveillance.org/ViewArticle.aspx?Articleld=19822

Article published on 24 March 2011

Efforts have been ongoing since 1996 to strengthen tuberculosis (TB) surveillance in Europe, starting with the launch of the EuroTB initiative. We present TB surveillance data for the Member States of the European Union (EU) and of the European Economic Area (EEA) for the latest reporting year (2009), highlighting key areas of epidemiological and programmatic focus. Despite a sustained decline of TB notifications at EU/ EEA level, several aspects of TB control can still be improved.

\section{The role of surveillance in} tuberculosis control in Europe

Surveillance is a basic component in the control and elimination of tuberculosis (TB) in Europe, and is one of the key areas of the Framework Action Plan to Fight TB in the European Union (EU) [1]. To attain a reliable surveillance system across Europe, a highly standardised data reporting system is needed. Persistent differences between national case definitions and reporting systems have, in the past, hampered the analysis of time trends, identification of population groups with high incidence and data comparability across the EU and the European Economic Area (EEA) and worldwide.

Efforts to standardise TB case reporting have been ongoing since at least 1996 in Europe [2]. The year 2011 marks the 15th anniversary of EuroTB, the European tuberculosis surveillance network. It was initiated as a project funded by the European Commission, housed and co-funded by Institut de Veille Sanitaire (the French Institute of Public Health Surveillance). Since 2008, EuroTB has been jointly coordinated by the European Centre for Disease Prevention and Control (ECDC) and the World Health Organization (WHO) Regional Office for Europe. The objective of this TB surveillance system has been to collect, validate, analyse and disseminate standardised high quality TB notification data covering the 53 countries of the WHO European Region and Liechtenstein. EuroTB had expanded the case-based surveillance towards participation of all countries from the WHO European Region, however after the transition of EuroTB to ECDC, the case-based surveillance remained only for the 30 EU/EEA countries. Designated national disease surveillance institutions are responsible for reporting the data to the European level through a joint data collection entry point. For EU/EEA countries, data are transmitted, validated and processed using The European Surveillance System (TESSy) run by ECDC, while data from all other countries are handled by the Centralized Information System for Infectious Diseases (CISID) run by the WHO Regional Office for Europe.

Over the past three years, an increasingly complete and reliable picture of the TB situation in the EU/ EEA and Europe has been achieved. In this article we present an overview of the most recent data reported in the latest Joint Annual TB surveillance Report for Europe [3] specifically for the 30 EU/EEA Member States, and highlight key areas of epidemiological and programmatic focus that represent pillars in TB control and thus benefit from a close and reliable monitoring at both national and regional level. At the start of the EuroTB, only 16 Member States of the EU/EEA were able to report case-based data to supranational level. After a constant increase in the number of countries reporting case-based data to European level, all 30 EU/EEA countries reported case-based TB surveillance data for 2007 and due to administrative constraints on the two last data collection rounds, Liechtenstein was not able to report to TESSy. The targets mentioned in the article are those endorsed by EU/EEA Member States in the follow-up to the Action Plan to Fight TB in the European Union [1].

\section{Current situation of tuberculosis in the European Union / European Economic Area Notifications}

In 2009, 79,665 TB cases were reported by the $27 \mathrm{EU}$ countries, Iceland and Norway, a decrease of 3,635 (4.5\%) cases compared with 2008. The decrease between 2007 and 2008 has been $1.4 \%$. 
Over $75 \%$ of cases occurred in the seven countries (two high-incidence countries and five low-incidence countries) that reported 3,000 cases or more (France, Germany, Italy, Poland, Romania, Spain and United Kingdom). The overall notification rate in 2009 was 15.8 per 100,000 population, the lowest number since the start of EuroTB in 1995 (Table) [5]. The cutoff point for high incidence countries in EU settings of 20 per 100,000 has been adopted by the Wolfeheze group in the 2002 report [4]. Despite a decrease in notification rates of $4.5 \%$ since 2008 , some countries still record high incidence with rates above 20 per 100,000 reported in Romania (108.2), Lithuania (62.1), Latvia (43.2), Estonia (30.7), Bulgaria (38.3), Portugal (27.0) and Poland (21.6). Seventy-nine percent of the cases were reported as new, while $13 \%$ were reported to have been previously treated. A new case (never treated) was defined as a case who had never previously received drug treatment for active TB, or who had received anti-TB drugs for less than one month. Belgium, Denmark, Ireland and United Kingdom used "never diagnosed" as proxy. As many as 6,327 notified cases $(7.9 \%)$ had no information on previous TB history, suggesting suboptimal surveillance in the reporting countries.

\section{Drug resistance}

In 2009, resistance to at least one first-line drug was reported for $3,870(14.1 \%)$ of 27,487 (35.7\% of all reported cases) tested cases in 28 EU/EEA countries: Austria, Belgium, Bulgaria, Cyprus, Czech Republic, Denmark, Estonia, Finland, France, Germany, Greece, Hungary, Iceland, Ireland, Italy, Latvia, Lithuania, Luxembourg, Malta, the Netherlands, Norway, Portugal, Romania, Slovakia, Slovenia, Spain, Sweden, United Kingdom while unknown drug susceptibility testing results were indicated for 13,336 (34.8\%) of 38,312 culture-positive cases in 25 of these $28 \mathrm{EU} /$

\section{TABLE}

Tuberculosis cases notification rates per 100,000 population, 1995 and 2009, EU/EEA ( $\mathrm{n}=30$ countries)

\begin{tabular}{|c|c|c|c|c|}
\hline Country & Total cases 1995 & $\begin{array}{r}\text { Notification rate per } \\
100,000 \text { population } 1995 \\
\text { (all cases) }\end{array}$ & Total cases 2009 & $\begin{array}{r}\text { Notification rate per } \\
100,000 \text { population } 2009 \\
\text { (all cases) }\end{array}$ \\
\hline Austria & 1,383 & 17.4 & 707 & 8.5 \\
\hline Belgium & 1,380 & 13.6 & 1,020 & 9.6 \\
\hline Bulgaria & 3,245 & 37.0 & 2,911 & 38.3 \\
\hline Cyprus & 36 & 5.6 & 55 & 6.9 \\
\hline Czech Republic & 1,851 & 17.9 & 702 & 6.7 \\
\hline Denmark & 448 & 8.6 & 329 & 6.0 \\
\hline Estonia & 608 & 42.0 & 411 & 30.7 \\
\hline Finland & 654 & 12.8 & 419 & 7.9 \\
\hline France & 8,723 & 14.7 & 5,308 & 8.2 \\
\hline Germany & 12,198 & 15.0 & 4,432 & 5.4 \\
\hline Greece & 939 & 8.9 & 586 & 5.2 \\
\hline Hungary & 4,339 & 42.0 & 1,448 & 14.4 \\
\hline Iceland & 12 & 4.5 & 9 & 2.8 \\
\hline Ireland & 458 & 12.7 & 472 & 10.6 \\
\hline Italy & 5,225 & 9.2 & 3,877 & 6.5 \\
\hline Latvia & 1,541 & 61.6 & 977 & 43.2 \\
\hline Liechtenstein & NA & NA & NA & NA \\
\hline Lithuania & 2,362 & 64.8 & 2,081 & 62.1 \\
\hline Luxembourg & 32 & 7.9 & 27 & 5.5 \\
\hline Malta & 10 & 2.7 & 44 & 10.6 \\
\hline Netherlands & 1,619 & 10.5 & 1,160 & 7.0 \\
\hline Norway & 236 & 5.4 & 363 & 7.6 \\
\hline Poland & 15,959 & 41.4 & 8,236 & 21.6 \\
\hline Portugal & 5,577 & 55.7 & 2,871 & 27.0 \\
\hline Romania & 23,271 & 102.5 & 23,267 & 108.2 \\
\hline Slovakia & 1,537 & 28.7 & 506 & 9.3 \\
\hline Slovenia & 525 & 26.4 & 188 & 9.3 \\
\hline Spain & 8,764 & 22.3 & 7,592 & 16.6 \\
\hline Sweden & 564 & 6.4 & 627 & 6.8 \\
\hline United Kingdom & 6,161 & 10.6 & 9,040 & 14.8 \\
\hline Total EU/EEA & 109,657 & 22.7 & 79,665 & 15.8 \\
\hline
\end{tabular}

EU/EEA: European Union/European Economic Area; NA: data not available. 
EEA countries (no unknown drug susceptibility testing results reported by Iceland, and excluding Italy and Poland due to lack of data). The proportion of cases with multidrug-resistant tuberculosis (MDR TB) in the 28 countries mentioned above was $5.3 \%$, a 0.7 percentage point decrease compared with 2008 and 2009, a 2.2 percentage point decrease compared with 2007 and 2008 [6], with the Baltic States and Romania reporting the highest proportions $(17.4 \%-28.0 \%$ and $11.2 \%$, respectively).

\section{Childhood tuberculosis}

The case notification rate of TB in children, especially infants, is a measure of the level of transmission in the community. In 2009, cases in children (under 15 years of age) accounted for $4.2 \%$ of all notified cases. Nearly all countries have experienced a decline or stabilisation at low levels in paediatric notification rates since 2005, suggesting low levels of transmission in the general population. In Bulgaria, Latvia, Lithuania and Romania, however, rates among children have remained high (12.9-29.6 per 100,000 child population) in 2009 and have increased in Bulgaria since 2000 (11.4-20.6 per 100,000$)$. Although rates in children were low in Belgium, Finland, Germany, the Netherlands, Slovenia, Sweden (less than 10 per 100,000), some increase in paediatric notifications was recorded between 2008 and 2009 in these countries (Belgium: from 2.9 to 3.6; Finland: from 0.4 to 0.8 ; Germany: from 1.1 to 1.3; the Netherlands: from 1.7 to 2.0; Slovenia: from 1.1 to 2.1; Sweden: from 1.8 to 2.1 ).

\section{Bacteriological confirmation of cases}

The proportion of bacteriologically confirmed TB cases remains sub-optimal in the EU/EEA countries, with only seven countries achieving the target of $80 \%$ culture-confirmation among new pulmonary TB cases, as reflects the approach to use only new cases, adopted in the monitoring framework endorsed by the Member

\section{FIGURE}

Treatment success rate among native and foreign confirmed tuberculosis cases, Europe, 2008 ( $\mathrm{n}=24$ countries)

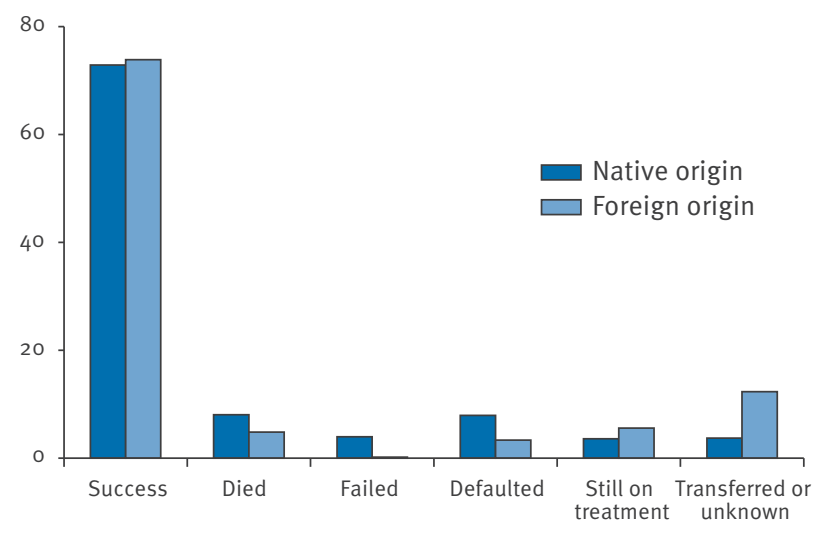

The geographical origin of tuberculosis cases is classified as native or foreign according to the place of birth (born in the country or foreign-born) or, if unavailable, according to citizenship (citizen/non-citizen).
States in the document A follow-up to the Framework Action Plan to Fight Tuberculosis in the European Union [7]. This consensus was reached after several months of consultation including discussion with the experts in the European Reference Laboratory Network for TB. Again with this article we try to achieve consistency of messages with the Annual Surveillance Report and ongoing EU standard approaches for monitoring.

Confirmation by culture was reported for $57.8 \%$ of all cases, whereas the culture result was reported as unknown for as many as $28.1 \%$ of the cases. The levels in culture confirmation differed widely across countries (range: $44 \%-100 \%$ ) and data were not complete for five countries.

High culture-coverage $(75 \%$ or more of cases with known culture result for Mycobacterium tuberculosis complex among all TB cases) was reported by eight countries and an additional eight countries showed an improvement in culture confirmation between 2005 and 2009. There were however some discouraging trends, with a decline in the proportion of culture confirmed cases in five countries from 2008 to 2009.

Overall, $78 \%$ of the cases were reported as pulmonary TB with or without extrapulmonary involvement. Sputum smear-positive rates were lower than five cases per 100,000 population in 21 countries in the last three years. The rates were consistently higher than 10 per 100,000 in the Baltic States, Bulgaria, Portugal and Romania.

\section{Treatment outcome}

For the 2008 cohort of culture-confirmed pulmonary TB cases, the overall treatment success rate was $72.8 \%$, with four countries reporting more than $85 \%$ treatment success (Malta, Portugal, Slovakia and Sweden). Compared with the situation in 2008 , treatment success decreased slightly among culture-confirmed pulmonary TB cases of foreign origin (from $75.7 \%$ to $73.8 \%$ ) and in cases of national origin (from $73.4 \%$ to $72.8 \%)$. A higher proportion of cases of national origin died compared with those of foreign origin.

Among previously untreated cases, $78.1 \%$ were cured, $6.7 \%$ died, $1.8 \%$ failed, $5.4 \%$ defaulted from treatment, $2.9 \%$ were still on treatment, and $5.2 \%$ were transferred or had an unknown outcome. Among countries with more than 20 previously untreated laboratory-confirmed pulmonary cases, success rates varied widely from $40.5 \%$ in Denmark to $87.4 \%$ in Sweden. In 1991, all WHO member states adopted a World Health Assembly (WHA) resolution setting two targets for global TB control to be reached by the year 2000: to detect at least $70 \%$ of all new infectious cases arising each year, and to cure at least $85 \%$ of those detected.

Six countries achieved treatment success in $85 \%$ or more of cases in this category: Bulgaria, $84.9 \%$, the Netherlands, $85.0 \%$, Slovakia, $87.0 \%$, Portugal, $87.3 \%$, 
Sweden, $87.4 \%$ and Malta, 92.3\%. Treatment success rates below $75 \%$ were associated with a high loss to follow-up (defaulted, transferred or unknown: 4.5\%$57.5 \%$ ). The higher proportion of defaulters among the native population would warrant further analysis, however, data presently available in TESSy are not sufficient to allow such an analysis.

\section{Conclusions}

The latest data reported for TB in the EU/EEA countries provide a comprehensive picture of the overall epidemiological situation in the region, with some limitations in terms of data comparability due to differences among surveillance systems in the Member States. This comprehensive epidemiological picture is the result of 15 years of collaborative efforts that began with the launch of EuroTB in 1996.

Despite limitations related to underreporting for selected variables (i.e culture confirmation, HIV status, among others), it is evident that the current reporting system could potentially support a thorough assessment of TB control in the EU/EEA on the basis of the indicators proposed in the follow-up to the Framework Action Plan to Fight TB in the EU [7]. Such an analysis is planned for the upcoming 2012 report. A number of the epidemiological and operational indicators included in the monitoring framework can be directly measured and calculated using the data collected in the current surveillance system.

It is also worth mentioning that despite a sustained average decline of the TB epidemic recorded in the EU/ EEA this is mainly attributable to the decline recorded in the high- and intermediate-burden countries. In addition several areas in need of strengthening like contact tracing, risk group management, laboratory data monitoring, treatment outcome reporting, were identified through the analysis of data.

Two crucial weaknesses in TB control in the EU/EEA are worth highlighting. The proportion of bacteriologically confirmed TB cases remains suboptimal, with only seven Member States achieving the target of $80 \%$ culture-confirmation among new pulmonary TB cases. This impedes the rapid detection of resistance and rapid provision of effective treatment to patients, thus preventing prompt interruption of transmission.

Although the number of countries achieving the target of $85 \%$ treatment success has doubled since 2008 , from a Treatment Outcome Monitoring perspective, the overall treatment success rate in the EU/EEA has not improved, furthermore, the rates are marginally decreasing (79.5\% to $78.1 \%$ ) between the 2007 and 2008 cohorts [8].

The potential for improving TB control in the EU/EEA therefore remains and the current TB surveillance setup, benefiting from 15 years of joint coordination, can provide direction and guidance in progressing towards TB elimination.
References

1. European Centre for Disease Prevention and Control (ECDC). Framework action plan to fight tuberculosis in the European Union. Stockholm:ECDC; Feb 2008. Available from: http:// www.ecdc.europa.eu/en/publications/Publications/0803_ SPR_TB_Action_plan.pdf

2. Schwoebel V, Rieder HL, Watson JM, Raviglione MC. Surveillance of tuberculosis in Europe. Euro Surveill. 1996;1(1):pii=200. Available from: http://www. eurosurveillance.org/ViewArticle.aspx?Articleld=200

3. European Centre for Disease Prevention and Control (ECDC) / World Health Organization Regional Office for Europe. Tuberculosis surveillance in Europe 2009. Stockholm: ECDC; Mar 2011. Available from: http://www.ecdc.europa.eu/en/ publications/Publications/1103_TB_SUR_2009.pdf

4. Broekmans JF, Migliori GB, Rieder HL, Lees J, Ruutu P, Loddenkemper R, et al. European framework for tuberculosis control and elimination in countries with a low incidence. Recommendations of the World Health Organization (WHO), International Union Against Tuberculosis and Lung Disease (IUATLD) and Royal Netherlands Tuberculosis Association (KNCV) Working Group. Eur Respir J. 2002;19(4):765-75.

5. European Commission / World Health Organization Regional Office for Europe. Tuberculosis cases reported in 1995. [Accessed 23 Mar 2011]. Available from: http://ecdc.europa. eu/en/publications/Publications/SUR_TB_EuroTB_Annual_ report_1995.pdf

6. European Centre for Disease Prevention and Control (ECDC) / World Health Organization Regional Office for Europe. Tuberculosis surveillance in Europe 2008. Stockholm: ECDC; Mar 2010. Available from: http://www.ecdc.europa. eu/en/publications/Publications/1003_SUR_tuberculosis_ surveillance_in_europe_2008.pdf

7. European Centre for Disease Prevention and Control (ECDC). Progressing towards TB elimination. A follow-up to the Framework Action Plan to Fight Tuberculosis in the European Union. Stockholm:ECDC; Nov 2010. Available from: http://www. ecdc.europa.eu/en/publications/Publications/101111_SPR Progressing_towards_TB_elimination.pdf

8. Manissero D, Hollo V, Huitric E, Ködmön C, Amato-Gauci A. Analysis of tuberculosis treatment outcomes in the European Union and European Economic Area: efforts needed towards optimal case management and control. Euro Surveill. 2010;15(11): pii=19514. Available from: http://www. eurosurveillance.org/ViewArticle.aspx?Articleld=19514 